\title{
Why Microbiome Treatments Could Pay Off Soon
}

Effective interventions may come before all the research is in By Rob Knight

Today we are at an exciting threshold of biology. Advances in DNA sequencing, coupled with high-end computation, are opening a frontier in new knowledge. Obtaining genetic information and obtaining insight from it have never been cheaper. The potential for curing previously incurable diseases, including chronic ones, seems immense. If this sounds familiar, you might be thinking that you heard it 15 years ago, when the Human Genome Project was in full swing. Many feel that genomic medicine has not yet delivered on its promise. So what is different this time with the microbiome? For one thing, you cannot really change your genome, but each of us has changed our microbiome profoundly throughout our lives. We have the potential not just to read out our microbiome and look at predispositions but to change it for the better.

What is most exciting at this stage is that we have mouse models that let us establish whether changes in the microbiome are causes or effects of disease. For example, we showed in collaborative work with Jeffrey I. Gordon's laboratory at Washington University in St. Louis last year that transferring the microbes from an obese person into mice raised in a bubble with no microbes of their own resulted in fatter mice. Normally, germ-free mice exposed to a mouse with microbialbased obesity would themselves become obese, but we could design a microbial community taken from lean

\section{We have the potential not just to read out our microbiome and look at predispositions but to change it for the better.}

